\title{
Phase II Pilot Clinical Study of the Use of Uncaria tomentosa (Cat's Claw) Combined with Paullinia cupana (Guarana) for the Treatment of Symptoms in Terminal Cancer Patients
}

\author{
Lais Beltrão Magalhães, Fernando Fonseca, Daniel Cubero and Auro Del Giglio* \\ Medical School of the Fundação ABC (ABC Foundation), Santo André, Brazil; aurodelgiglio@gmail.com
}

\begin{abstract}
Previous studies in patients with advanced cancer have reported that Cat's claw dry extract (Uncaria tomentosa) and Guarana (Paullinia cupana) showed beneficial effects in patients with terminal cancer. Our objective is to investigate the combined action of these extracts in the control of symptoms in these patients. This prospective, uncontrolled pilot study evaluated the use of $100 \mathrm{mg}$ of $U$. tomentosa dry extract three times daily combined with $50 \mathrm{mg}$ of $P$. cupana dry extract twice daily in terminal patients with solid tumors who were ineligible for traditional therapeutic approaches. Patients had blood collected and answered questionnaires (EORTC QLQ-C30, FACIT-F, Chalder Fatigue Scale, HADS and PSQI-BR) before and after 3 and 6 weeks of treatment. Twenty-two volunteers were recruited; the mean age of the population was 68.3 years (range, $49-89$ years), $86.3 \%$ were men, and half had a Karnofsky Index less than or equal to $60 \%$. We observed improvement in fatigue, measured by the Chalder Scale $(p=0.0041)$, and nausea, measured by the EORTC QLQ-C30 scale $(p=0.0470)$, without significant weight loss $(p=0.3883)$ or influence on quality of life as measured by the FACIT-F questionnaire $(p=0.3375)$. Medications were well tolerated by most participants. No significant reduction in inflammatory parameters was observed during the treatment. The combination of $U$. tomentos $a$ and $P$. cupana extracts is safe and has potential benefits for the control of fatigue and nausea in terminal cancer patients.
\end{abstract}

Keywords: Cancer, Clinical Trial, Palliative Care, Paullinia cupana, Uncaria tomentosa

\section{Introduction}

Paullinia cupana (Sapindaceae), popularly known as Guarana, is a plant native to the Amazon basin. It contains purine alkaloids, such as caffeine, theophylline and theobromine, which are responsible for the stimulating effects of guarana ${ }^{1}$. The caffeine content in samples of guarana extract presents great variability, ranging from 9.52 to $36.71 \mathrm{mg} / \mathrm{g}$ of powder, and when compared to the dry extract of coffee, the caffeine concentration is about four times higher ${ }^{2}$.

The psychoactive properties of guarana are also attributed to tannins and saponins. Guarana exhibits antioxidant effects, even at very low concentrations
$(1.2 \mu \mathrm{g} / \mathrm{ml})$ it can inhibit lipid peroxidation. At high doses $(1000-2000 \mathrm{mg} / \mathrm{kg})$, it has no significant toxic or cytotoxic effects, even after prolonged exposure ${ }^{3}$. Antioxidant $^{4}$ and anti-inflammatory effects were also demonstrated in animals and produced a significant reduction in cytokine levels ${ }^{5}$.

In patients with breast cancer undergoing adjuvant chemotherapy, guarana demonstrated significant clinical activity in reducing fatigue compared to placebo ${ }^{6}$. Additionally, in an uncontrolled clinical pilot study, the use of guarana in a population with advanced oncological disease resulted in increased appetite, weight stabilization and reduced sleepiness ${ }^{7}$. 
U. tomentosa (Cat's claw) (Rubiaceae) is native to South America, a region comprising the Amazon river basin ${ }^{8}$. In Brazil, Cat's claw can be found in the states of Acre, Amapá, Amazonas and Pará 9 . The U. tomentosa extract has a variety of bioactive metabolites, including oxindole, indole, and pentacyclic alkaloids, quinovic acid glycosides, oxygenated triterpenes and procyanidins ${ }^{10}$.

The clinical use of $U$. tomentosa in a population with advanced oncologic diseases and beyond therapeutic possibility produced reduced fatigue evaluated by quality of life questionnaires. Stabilization of body weight and, in some cases, tumor growth, was also verified but without an objective response confirmation. The study showed no changes in the inflammatory markers levels (TNF- $\alpha$, IL-1, IL-6, ESR, CRP or $\alpha-1$ acid glycoprotein), and the results obtained were likely unrelated to the antiinflammatory action of the medication ${ }^{11}$.

Hence, this study was planned to evaluate the combined use of two extracts, $U$. tomentosa and $P$. cupana on symptoms associated with cancer in a group of patients with advanced solid tumors, previously treated and beyond therapeutic possibility. We analyzed the safety of this combination of extracts and specifically its influence on quality of life, fatigue, sleep quality, weight and inflammatory markers.

\section{Materials and Methods}

\subsection{Study Design}

This was a prospective, uncontrolled, unicentric pilot study. Recruitment and follow-up were performed at the clinical oncology outpatient clinics of Hospital Anchieta (Anchieta Hospital) in São Bernardo do Campo and Hospital Estadual Mario Covas (Mario Covas State Hospital) in Santo André, São Paulo state, both of which are linked to the Medical School of the Fundação ABC (ABC Foundation).

This study was approved by the Institutional Research Ethics Committee of the Medical School of the ABC Foundation. Patients were included after consenting to all study procedures, which was confirmed by signing the informed consent form.

\subsection{Inclusion and Exclusion Criteria}

Patients older than 18 years were included, with histologically proven solid tumors that had been previously treated and who had no therapeutic possibility according to their attending physician at the time of inclusion. Patients with a life expectancy of at least 2 months according to the judgment of the attending physician were included. The patients with following criteria were excluded: a) those with creatinine levels greater than twice the upper limit of normality; b) those with Alanine Aminotransferase (ALT) and Aspartate Aminotransferase (AST) levels greater than twice the upper limit of normality, (patients with liver disease with an increased AST and ALT levels greater than two and a halftimes the upper limit of normality were also excluded); c) direct bilirubin levels greater than one and a half times the upper limit of normality (patients with liver disease with an increased direct bilirubin levels greater than two and a half times the upper limit of normality were also excluded); d) patients who were in the gestational and/ or lactation period (women of childbearing age were included only if they had a negative quantitative BetaHuman Chorionic Gonadotropin (BHCG) test result); e) patients in current use of chemotherapy or other antineoplastic treatments with an anti-tumor therapeutic purpose (antalgic radiotherapy was not an exclusion criterion); f) patients with hypersensitivity to any of the components administered; and g) patients with a known clinical history of emotional disorders that could compromise their participation in the study.

The criteria for discontinuation of the study were as follows: a) adverse events of grade 3 or 4 according to the Common Terminology Criteria for Adverse Events (CTCAE-Version 4); b) the need to use concomitant medications prohibited with chemotherapy or hormone therapy; and c) nonadherence to treatment (use of less than $80 \%$ of the drug during the study period) and/or violation of the protocol.

After consent, a thorough evaluation of the clinical history of each patient and a complete physical examination was performed by the specialist investigators. At the same visit, biological material was collected to perform tests to identify alterations that could make it impossible to participate in the study. 
The protocol was designed with four visits, including one visit to determine eligibility. The eligibility evaluation was performed one week before inclusion $(\mathrm{T}=-1)$. The first visit $(\mathrm{T}=0)$ and the other two visits occurred every three weeks until week six of treatment. At each visit, a clinical examination and recording of the study variables were performed through the use of specific questionnaires and blood collection. The questionnaires used to assess the quality of life were the following: European Organization for the Research and Treatment of Cancer Quality of Life Questionnaire (EORTC QLQ-C30, version 3) ${ }^{12}$; Functional Assessment of Chronic Illness Therapy- Fatigue (FACIT-F, version $4)^{13}$; Hospital Anxiety and Depression Scale (HADS) ${ }^{14}$; Chalder Fatigue Scale ${ }^{15}$. Sleep quality was assessed through the Brazilian version of the Pittsburgh Sleep Quality Index (PSQI-BR) ${ }^{16}$.

Blood samples were collected every three weeks to evaluate hematological and biochemical profiles, to detect possible renal and/or hepatic toxicity reactions and to investigate inflammatory markers (PCR, Il-1, Il6, $\alpha-1$ acid glycoprotein, VHS and TNF- $\alpha$ ).

Adverse events were recorded according to the International Criteria for Notification of Adverse Events, version 4.0 (CTCAE-V4.0).

\subsection{Medications}

One of the medications administered was $U$. tomentosa dry extract (batch number 160907), $100 \mathrm{mg}$ tablets, standardized to a total alkaloid concentration (corresponding to mitraphylline) of $5.0 \pm 0.5 \%$, as measured by chromatography. The extract was procured from Herbarium Laboratory (Colombo, Paraná, Brazil) and was derived from the root cortex of the plant. One tablet was given three times a day, every day, according to the package insert.

The other medication administered was the dried extract of $P$. cupana (lot number 11.20111 1), $50 \mathrm{mg}$ capsules, purchased from CatedralIndústria Farmacêutica (Nova Pampulha, Vespaziano, Minas Gerais state, Brazil). Corn starch was used as an excipient. The preparation of guarana had a $\mathrm{pH}$ of 4.83 ( $10 \%$ solution in water), water content of $3.9 \%$, tannin concentration of $1.7 \%$ and caffeine concentration of
$6.62 \%$. The caffeine concentration was used to standardize the solution. A $50 \mathrm{mg}$ capsule was administered twice daily, if administration failure occurred for any reason, patients were advised not to double the next dose. In case of possible adverse effects, dose reduction (reduction of one dose) was advised; if symptoms persisted, the medication was withdrawn. Adherence to treatment was assessed at each visit by the return of medication packages.

\subsection{Laboratory Tests}

Approximately $20.0 \mathrm{ml}$ of whole blood was collected from all participants at each visit. The blood sample was distributed in tubes with and without the anticoagulant Ethylenediaminetetraacetic Acid (EDTA). EDTAcontaining tubes were used for complete blood count using a flow cytometer, ABX Pentra 120 (HORIBA ${ }^{\oplus}$, Japan). The results were confirmed on smear slides fixed with a Leishman fixative. The blood sedimentation at 1 hour was evaluated by the Wintrobe method.

Blood in the EDTA-free tubes was used for biochemical testing. Levels of creatinine, AST, ALT, alkaline phosphatase, Gamma-Glutamyl Transpeptidase (GGT) and total and fractionated bilirubin levels were obtained by an automated enzymatic-colorimetric assay obtained from Roche ${ }^{\circledast}$ using a Cobas ${ }^{\circledast} 6000$ spectrophotometer. TNF- $\alpha$, IL- 6 and PCR were evaluated by a competitive immunoassay obtained from Siemens ${ }^{\circledast}$ by reading chemiluminescence using an Immulite 1000 luminometer. Alpha-1 acid glycoprotein levels were measured by immunoturbidimetry. All tests were performed twice, according to the good clinical practice standards adopted by the Laboratory of Clinical Tests of the Medical School of the ABC Foundation.

\subsection{Statistical Tests}

We used the nonparametric Kruskal-Wallis test to evaluate the presence of significant differences between the questionnaire scores and laboratory values obtained from patients at the beginning of the study and after 3 and 6 weeks of treatment. We considered p-values lower than 0.05 to be statistically significant. GraphPad Prism version $6 \mathrm{~d}$ was used for all statistical calculations (GraphPad Software, La Jolla, CA). 


\section{Results}

\subsection{Study Population}

In the period between May 2017 and January 2018, 26 patients were recruited; of these patients, 10 (38\%) were included from the Anchieta Hospital in São Bernardo do Campo - SP, and 16 (62\%) were included from the Mario Covas State Hospital in Santo André - SP. Among the patients recruited, 12 completed all three consultations provided in the initial protocol, seven patients completed two consultations, and seven patients had only one consultation.

Table 1. Table of socio-demographic characteristics

\begin{tabular}{lr}
\hline \multicolumn{1}{c}{ Variable } & Data \\
\hline Mean age (range) (y) & $69.2(49-90)$ \\
Sex & \\
Male & $88.4 \%(23)$ \\
Female & $11.5 \%(3)$ \\
Ethnicity & \\
Black & $35 \%(9)$ \\
White & $65.4 \%(17)$ \\
Asian & 0 \\
Primary cancer site/cancer type & \\
Lung & \\
Esophagus & $12 \%(3)$ \\
Prostate & $8 \%(2)$ \\
Rectum & $19 \%(5)$ \\
Stomach & $15 \%(4)$ \\
Liver & $4 \%(1)$ \\
Kidney & $4 \%(1)$ \\
Central nervous system & $4 \%(1)$ \\
Cervix & $4 \%(1)$ \\
Ovary & $4 \%(1)$ \\
Oropharynx & $4 \%(1)$ \\
Colon & $8 \%(2)$ \\
Melanoma & $8 \%(2)$ \\
Karnofsky Performance Status & $4 \%(1)$ \\
100\% & \\
$80 \%$ & \\
70\% & \\
$\leq 60 \%$ & 0 \\
& \\
\hline & \\
\hline
\end{tabular}

The socio-demographic characteristics are listed in Table 1 . The mean age of the population was 69.2 years (range, 49-90 years). The distribution between men and women was not balanced, with 23 (88.4\%) men and 3 (11.5\%) women; most of them were white
( $\mathrm{n}=17 ; 65.4 \%)$, and $9(35 \%)$ were black. The distribution among solid tumors was quite heterogeneous, with the most prevalent being prostate, rectal and lung cancer (19\%, 15\% and $12 \%$, respectively). More than half of the sample (54\%) had a Karnofsky Performance Status Index less than or equal to $60 \%$.

\subsection{Results of the Questionnaires}

When we evaluated fatigue using the Chalder Scale, we observed that the patients showed an improvement of symptoms during the treatment $(\mathrm{p}=0.0012)$. We observed that the score in the FACIT-F questionnaire presented stability during the treatment period $(p=0.2080)$, which demonstrates a tendency to maintain quality of life scores (Figure 1).
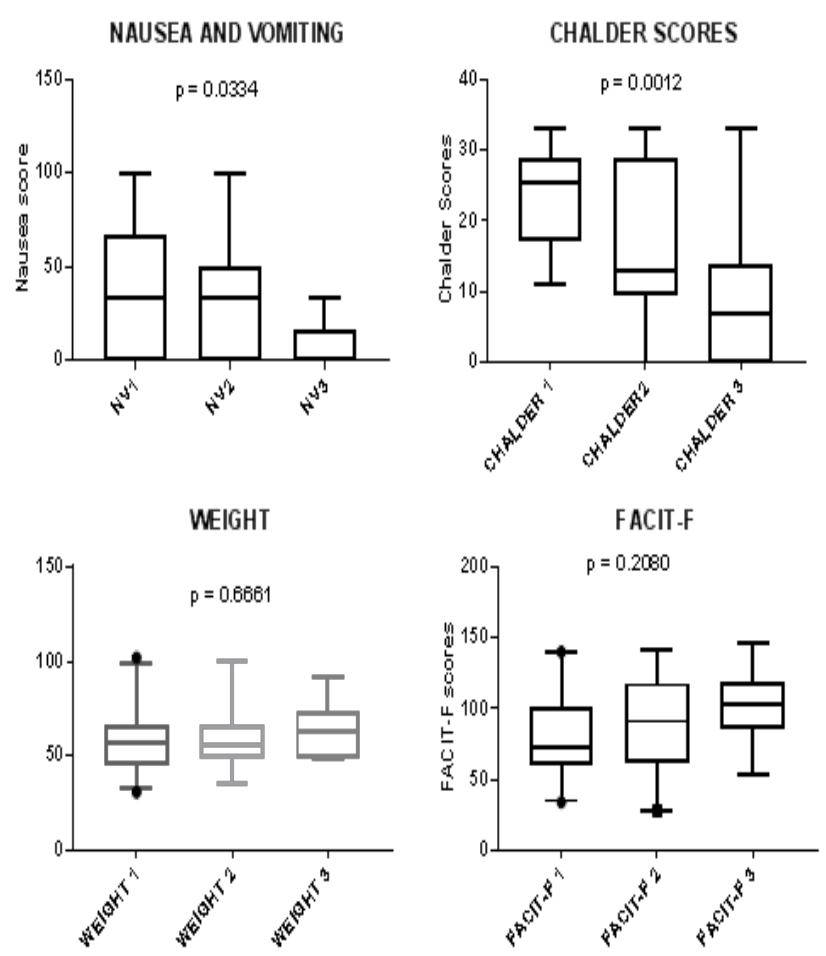

Figure 1. Scores for the FACIT-F Scale, Chalder Fatigue Scale, weight curve during treatment, and the nausea and vomiting symptoms scale of the European Organization for the Research and Treatment of Cancer Quality of Life Questionnaire (EORTC QLQ-C30) during the treatment period. A confidence interval of $95 \%$ and $p$-values less than 0.05 were considered statistically significant. The $p$-value corresponds to the comparison of the scores before and during the use of the medication by the Kruskal-Wallis test. 
It was observed through the EORTC QLQ-C30 that the nausea curve decreased throughout the treatment $(\mathrm{p}=0.0334)$. Patients' weight also remained stable during the treatment period $(\mathrm{p}=0.6661)$ (Figure 1). Other symptoms, including insomnia, pain, reduced appetite and aspects of functional, physical, emotional and cognitive well-being, did not show significant differences throughout the treatment.

\subsection{Laboratory Parameters}

Laboratory test results (complete blood count, liver function, ALT, AST, alpha-fetoprotein, GGT and bilirubin) did not change significantly over the course of treatment. We did not observe a significant decrease in inflammatory parameters (CRP, $\alpha-1$ acid glycoprotein or IL-6) during the study. TNF- $\alpha$ increased significantly during the study (Figure 2).
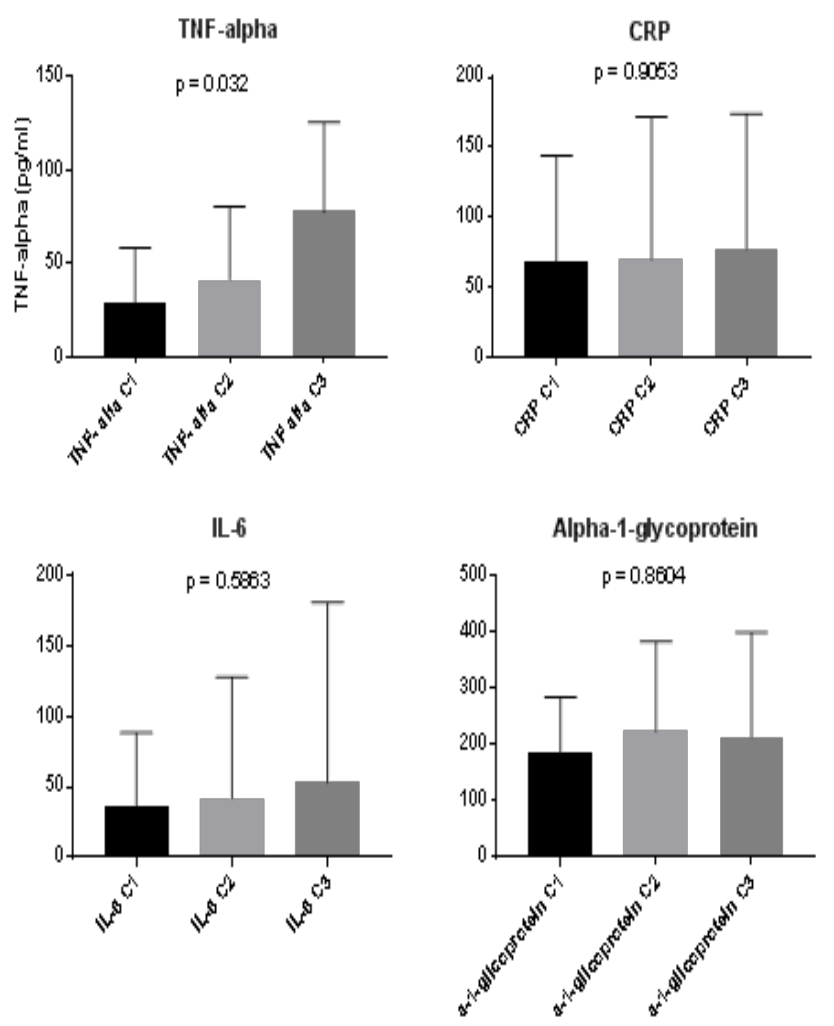

Figure 2. Inflammatory parameters and blood levels during treatment with $U$. tomentosa and $P$. cupana. Confidence interval of $95 \%$ and $p$-values less than 0.05 were considered statistically significant. The p-value corresponds to the comparison of the scores before and during the use of the medication by the Kruskal-Wallis test.

\subsection{Adverse Events}

Adverse events are listed in Table 2. There were few grade 3 or 4 adverse events on the CTCAE scale. Patients who had grade 3 toxicity to GGT and alkaline phosphatase already had these levels at inclusion and maintained stable levels throughout the treatment. One patient presented grade 3 diarrhea at the second visit, was treated with an antibiotic, and the symptom was resolved. Patients who presented grade 3 insomnia exhibited this symptom since recruitment. One patient with grade 3 lower limb pain presented cervical cancer with disease in the pelvic lymph nodes and had difficulty controlling radiating pain due to the progression of the disease. A patient with a grade 3 urinary tract infection hospitalized for treatment showed improvement. A patient with grade 3 lumbar spine pain due to metastatic bone disease experienced improvement in pain after palliative radiotherapy.

Among the patients included, seven patients were lost to follow-up after the first consultation. One patient reported having interrupted the medication due to worsening nausea and vomiting at the first visit and presented grade 3 constipation and symptoms of grade 1 intestinal subocclusion, was assessed and hospitalized before the second visit, and presented a worsening of intestinal subocclusion symptoms by progression of peritoneal carcinomatosis. Two patients, who at the first consultation referred to grade 1 nausea, complained of worsening nausea after starting the medication and stopped using it, opting out of the protocol. One patient with a locally advanced prostate tumor and metastatic lesion presented digestive hemorrhage probably related to rectal invasion due to prostate neoplasm. Three patients were hospitalized for clinical-infectious complications related to the underlying neoplasia and died after the first consultation.

Two patients died before the third visit due to complications related to central nervous system disease. One patient presented grade 2 mental confusion at the second visit, was referred for evaluation and diagnosed with grade 3 pneumonia and died of septic shock. One patient who reported a worsening of nausea at the second visit (grade 2 nausea) was evaluated with a possible intestinal subocclusion and was hospitalized, and they died due to this cause. 
Phase II Pilot Clinical Study of the Use of Uncaria tomentosa (Cat's Claw) Combined with Paullinia cupana (Guarana) for the Treatment of Symptoms in Terminal Cancer Patients

Table 2. Adverse events that occurred during the study

\begin{tabular}{|c|c|c|c|c|c|c|c|c|}
\hline & \multicolumn{2}{|c|}{ Grade 1} & \multicolumn{2}{|c|}{ Grade 2} & \multicolumn{2}{|c|}{ Grade 3} & \multicolumn{2}{|c|}{ Grade 4} \\
\hline & $\mathbf{N}$ & $\%$ & $\mathbf{N}$ & $\%$ & $\mathbf{N}$ & $\%$ & $\mathbf{N}$ & $\%$ \\
\hline \multicolumn{9}{|l|}{ Gastrointestinal disorders } \\
\hline Nausea & 7 & $27 \%$ & 12 & $46 \%$ & & & & \\
\hline Vomiting & 10 & $38 \%$ & 1 & $8 \%$ & & & & \\
\hline Abdominal pain & 4 & $15 \%$ & & & & & & \\
\hline Constipation & 9 & $35 \%$ & 5 & $19 \%$ & 1 & $4 \%$ & & \\
\hline Epigastric pain & 1 & $4 \%$ & 1 & $4 \%$ & & & & \\
\hline Diarrhea & 2 & $8 \%$ & 1 & $4 \%$ & 1 & $4 \%$ & & \\
\hline Anal pain & 1 & $4 \%$ & & & & & & \\
\hline Rectal hemorrhage & 2 & $8 \%$ & & & & & & \\
\hline Partial bowel obstruction & 2 & $8 \%$ & & & & & 1 & $4 \%$ \\
\hline \multicolumn{9}{|l|}{ Blood disorders } \\
\hline Anemia & 13 & $50 \%$ & 5 & $19 \%$ & 1 & $4 \%$ & & \\
\hline \multicolumn{9}{|l|}{ Metabolic disorders } \\
\hline AST increase & 2 & $8 \%$ & & & & & & \\
\hline ALT increase & 2 & $8 \%$ & & & & & & \\
\hline AP increase & 2 & $8 \%$ & 4 & $15 \%$ & 2 & $8 \%$ & & \\
\hline GGT increase & 7 & $27 \%$ & 2 & $8 \%$ & 5 & $19 \%$ & & \\
\hline Bilirubin & & & 2 & $8 \%$ & & & & \\
\hline Creatinine increase & 2 & $8 \%$ & & & & & & \\
\hline \multicolumn{9}{|l|}{ General and other disorders } \\
\hline Fatigue & 6 & $23 \%$ & 13 & $50 \%$ & 7 & $27 \%$ & & \\
\hline Insomnia & 7 & $27 \%$ & 6 & $23 \%$ & 2 & $8 \%$ & & \\
\hline Sleepiness & 3 & $12 \%$ & & & & & & \\
\hline Anorexia & 12 & $46 \%$ & 5 & $19 \%$ & 1 & $4 \%$ & & \\
\hline Mental confusion & 2 & $8 \%$ & & & & & & \\
\hline Dyspnea & 7 & $27 \%$ & 1 & $4 \%$ & & & & \\
\hline Lower limb pain & 2 & $8 \%$ & 3 & $12 \%$ & 1 & $4 \%$ & & \\
\hline Lower limb swelling & 3 & $12 \%$ & & & & & & \\
\hline Pneumonia & & & 1 & $4 \%$ & 2 & $8 \%$ & & \\
\hline Urinary tract infection & & & 4 & $15 \%$ & & & & \\
\hline Bone pain & 2 & $8 \%$ & 5 & $19 \%$ & & & & \\
\hline Back pain & & & & & 1 & $4 \%$ & & \\
\hline Arthralgia & 2 & $8 \%$ & 1 & $4 \%$ & 1 & $4 \%$ & & \\
\hline Muscle weakness of the lower limbs & & & 2 & $8 \%$ & 1 & $4 \%$ & & \\
\hline Myalgia & 1 & $4 \%$ & & & & & & \\
\hline Headache & 2 & $8 \%$ & & & & & & \\
\hline
\end{tabular}

AST, aspartate aminotransferase; ALT, alanine aminotransferase; AP, alkaline phosphatase; GGT, gamma-glutamyl transferase. 


\section{Discussion}

This study combined two herbs $U$. tomentosa and $P$. cupana that individually produced beneficial clinical effects in terminal cancer patients ${ }^{7,11}$. The effect and safety of the combined use of these substances in individuals with advanced disease with no further therapeutic options, however, is unknown.

In fact, previous studies conducted by our group have demonstrated interesting effects of these phytotherapeutics alone related to the control of symptoms in cancer patients. A previous phase II, double-blind, placebo-controlled crossover study evaluated the effect of high doses of guarana (50 mg twice daily) on fatigue related to chemotherapy ${ }^{17}$. In another pilot study using guarana in a population with advanced oncological disease, guarana also demonstrated benefits of increased appetite, weight stabilization, and reduced sleepiness ${ }^{7}$. Additionally, the isolated clinical use of $U$. tomentosa in a population with advanced oncologic disease and without therapeutic possibility also produced reduced fatigue, evaluated by quality of life questionnaires, associated with body weight stabilization ${ }^{11}$.

In this pilot study, we observed that this combination produced encouraging results in terms of weight maintenance and quality of life in addition to improved fatigue and decreased nausea and vomiting. Additionally, the combined use of extracts of $U$. tomentosa and $P$. cupana appears to be safe for the studied population; there were few grade 3 or 4 adverse effects.

Cancer-related fatigue is defined as a subjective and persistent sensation of physical, mental, and cognitive fatigue or exhaustion related to cancer or cancer treatment, which is not commensurate with the tasks performed and interferes with daily activities. It occurs in 65 to $100 \%$ of patients with advanced disease ${ }^{17,18}$ and is one of the most important symptoms related to the reduction in the quality of life of cancer patients and can originate from the tumor activity, release of inflammatory cytokines, or immune response against the tumor, among other factors. However, in this study, as well as in a previous study on the use of $U$. tomentos $a^{11}$, we did not observe a decrease in inflammatory parameters together with the reduction of fatigue. It is possible that the beneficial effects observed in terms of reduced fatigue and decreased nausea and vomiting were not produced by the action of these herbal medicines on inflammatory pathways.
This study has important limitations. The studied population was small and unbalanced in gender distribution and consisted of a heterogeneous group of patients, mostly elderly patients with different solid tumors and low performance status. As often happens in studies conducted with terminally ill patients, there were follow-up losses, often due to clinical complications related to the natural progression of advanced neoplasia ${ }^{11}$. This loss of follow-up may have biased our results, since the loss of more fragile patients from the study could have overestimated the beneficial effects of this combination of phytotherapeutic drugs. In addition, the absence of a control group makes it impossible to estimate how much of the clinical effects obtained were due to the placebo effect. Furthermore, the anti-nusea effects we observed with this combination need to be compared in future studies with other conventional and phytoterapic anti-nausea medications such as 5HT3 antagonists and ginger, respectively.

\section{Conclusion}

In conclusion, the combined use of $U$. tomentosa and $P$. cupana has been shown to be safe in patients with solid tumors who are ineligible for traditional therapeutic approaches. The beneficial effects on decreased fatigue and nausea and vomiting may be beneficial for this group of patients. Placebo-controlled studies involving a larger number of patients should be conducted to confirm these results.

\section{References}

1. Weckerle CS, Stutz MA, Baumann TW. Purine alkaloids in Paullinia. Phytochemistry. 2003; 64(3):735-42. Crossref.

2. Tfouni SAV, Camargo MCR, Vitorino SHP, Menegário TF, Toledo MCF. Contribuição do Guaraná em pó (Paullinia cupana) como fonte de cafeína na dieta. Revista de Nutrição. 2007; 20(1):63-8. Crossref.

3. Mattei R, Dias RF, Espinola EB, Carlini EA, Barros SB. Guarana (Paullinia cupana): toxic behavioral effects in laboratory animals and antioxidants activity in vitro. J Ethnopharmacol. 1998; 60(2):111-6. Crossref.

4. EspinolaEB, Dias RF, Mattei R, CarliniEA.Pharmacological activity of Guarana (Paullinia cupana Mart.) in laboratory animals. J Ethnopharmacol. 1997; 55(3):223-9. Crossref. 
5. Marcon R, Del Giglio A, Pianowski LF, Calixto JB. An analysis of the role of Paulinia cupana (Guaraná) in blood IL- $1 \mathrm{~b}$ and brain IL-6 levels as well as brain IL-6 and TNF-a mRNA expression in mice. J Clin Oncol. 2011; 29(15_ Suppl):e21002.

6. de Oliveira CMP, Riechelmann R, Martins LC, Hassan BJ, Casa FB, Del Giglio A. Guarana (Paullinia cupana) improves fatigue in breast cancer patients undergoing systemic chemotherapy. J Altern Complement Med. 2011; 17(6):505-12. Crossref. PMid:21612429

7. Palma CG, Lera AT, Lerner T, de Oliveira MM, de Borta TM, Barbosa RP, et al. Guarana (Paullinia cupana) improves Anorexia in patients with advanced cancer. J Diet Suppl. 2016; 13(2):221-31. Crossref. PMid:25695932

8. Zevallos-Pollito PA, Filho TM. Levantamento e caracterização de duas espécies do gênero Uncaria schreb. (Rubiaceae) correntes no Estado do Acre, Brasil. Ecol Apl. 2010; 9(1):19-30. Crossref.

9. Pereira RDC, Pinto J, Bertolucci SKV, Castro ED, Silva FG. Germinação, avaliação do ácido giberélico e posição do explante no alongamento in vitro de Uncaria guianensis (Aublet) Gmelin Rubiaceae (Unha-de-gato). Cien Agrotec. 2006; 30(4):637-42. Crossref.

10. Aquino R, De Simone F, Pizza C, Conti C, Stein ML. Plant metabolites. Structure and in vitro antiviral activity of quinovic acid glycosides from Uncaria tomentosa and Guettarda platypoda. J Nat Prod. 1989; 52(4):679-85. Crossref. PMid:2553871

11. de Paula LC, Fonseca F, Perazzo F, Cruz FM, Cubero D, Trufelli DC, et al. Uncaria tomentosa (cat's claw) improves quality of life in patients with advanced solid tumors. J
Altern Complement Med. 2015; 21(1):22-30. Crossref. PMid:25495394

12. Fayers P, Aaronson N, Bjordal K, Groenvold M, Curran D, Bottomley A. The EORTC QLQ-C30 scoring manual. Brussels: European Organization for Research and Treatment of Cancer; 2001. PMCid:PMC2363932

13. Peipert JD, Beaumont JL, Bode R, Cella D, Garcia SF, Hahn EA. Development and validation of the functional assessment of chronic illness therapy treatment satisfaction (FACIT TS) measures. Qual Life Res. 2014; 23(3):815-24. Crossref. PMid:24062239

14. Zigmond AS, Snaith RP. The hospital anxiety and depression scale. Acta Psychiatr Scand. 1983; 67(6):36170. Crossref.

15. Cho HJ, Costa E, Menezes PR, Chalder T, Bhugra D, Wessely S. Cross-cultural validation of the Chalder Fatigue Questionnaire in Brazilian primary care. J Psychosom Res. 2007; 62(3):301-4. Crossref. PMid:17324680

16. Buysse DJ, Reynolds CF, Monk TH, Berman SR, Kupfer DJ. The Pittsburgh Sleep Quality Index: a new instrument for psychiatric practice and research. Psychiatry Res. 1989; 28(2):193-213. Crossref.

17. Campos MP, Hassan BJ, Riechelmann R, Del Giglio A. Cancer-related fatigue: a practical review. Ann Oncol. 2011; 22(6):1273-9. Crossref. PMid:21325448

18. Baró E, Carulla J, Cassinello J, Colomer R, Mata JG, Gascón P, et al. Psychometric properties of the perform questionnaire: a brief scale for assessing patient perceptions of fatigue in cancer. Support Care Cancer. 2011; 19(5):65766. Crossref. PMid:20432046 PMCid:PMC3069331 